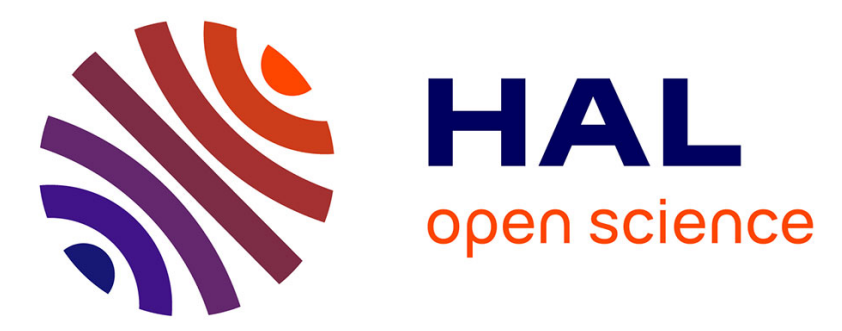

\title{
QoE-based performance evaluation of video transmission using RPL in the IoMT
}

\author{
Souhila Kettouche, Moufida Maimour, Lakhdar Derdouri
}

\section{To cite this version:}

Souhila Kettouche, Moufida Maimour, Lakhdar Derdouri. QoE-based performance evaluation of video transmission using RPL in the IoMT. 7th Mediterranean Congress of Telecommunications, CMT 2019, Oct 2019, Fès, Morocco. 10.1109/CMT.2019.8931382 . hal-02416167

\section{HAL Id: hal-02416167 \\ https://hal.science/hal-02416167}

Submitted on 17 Dec 2019

HAL is a multi-disciplinary open access archive for the deposit and dissemination of scientific research documents, whether they are published or not. The documents may come from teaching and research institutions in France or abroad, or from public or private research centers.
L'archive ouverte pluridisciplinaire HAL, est destinée au dépôt et à la diffusion de documents scientifiques de niveau recherche, publiés ou non, émanant des établissements d'enseignement et de recherche français ou étrangers, des laboratoires publics ou privés. 


\section{QoE-based Performance Evaluation of Video Transmission using RPL in the IoMT}

\author{
Souhila Kettouche \\ RELA $(C S)^{2}$, Larbi Ben M'hidi University \\ Oum El Bouaghi, Algeria \\ souhila.kettouche@gmail.com
}

\author{
Moufida Maimour \\ CNRS, CRAN, UMR 7039, France \\ Lorraine University, Nancy, France \\ moufida.maimour@univ-lorraine.fr
}

\author{
Lakhdar Derdouri \\ RELA $(C S)^{2}$,Larbi Ben M'hidi University \\ Oum El Bouaghi, Algeria \\ derdouril@yahoo.fr
}

\begin{abstract}
Internet of Things systems can not successfully realise the notion of ubiquitous connectivity of everything if they are not capable to truly include multimedia things. In this paper, we investigate the feasibility of video streaming using RPL (Routing Protocol for Low Power and Lossy Networks) by focusing on the impact of radio duty cycling on the quality of received video and the energy footprint of the network. To do so, we adopt a low complexity compression technique and use Cooja simulator to carry out our experiments. We use quality of service $(\mathrm{QoS})$ as well as quality of experience (QoE) metrics to evaluate the quality of the received video. We mainly show that RPL along with ContikiMAC, the Contiki default radio duty cycling do not allow to handle real time video transmission in the context of constrained networks. However low rates up to 35 frames per minute are still possible with an acceptable quality.
\end{abstract}

Index Terms-Internet of Multimedia Things (IoMT) ; RPL ; Radio Duty Cycling (RDC) ; QoE ; QoS ; Contiki OS.

\section{INTRODUCTION}

Technology advances foster the development of low-power and low-cost visual modules [1] that allow the emergence of Wireless Visual Sensor Networks (WVSN), a key building block of the Internet of Multimedia Things (IoMT). Visual data provide an added value to numerous applications especially those related to surveillance and monitoring. RPL [2] is the IETF standardised IPv6 Routing Protocol for low-power and lossy networks (LLNs) where directed-oriented acyclic graphs (DODAG) rooted at the sink are built. An objective function is used by each node to select its preferred parent toward the root node. Two objective functions namely, ETX [3] (default) and $O F O$ (hops number) are predefined.

Despite the fact that RPL almost meets the requirements of LLNs to handle scalar data routing, it still far from being able to allow real time streaming of video flows. Visual sensors with limited resources have to deal with large amount of data to capture, encode and transmit. These tasks result in significant power consumption while sensor nodes are equipped with limited batteries capacity. Authors of [4] considered video traffic routing using RPL and proposed a new objective function called Green-RPL in an attempt to minimise energy consumption while assuring a required quality of service (QoS). Simulation results show that Green-RPL outperforms $E T X$ and $O F O$ in terms of consumed energy, number of delivered packets and delay. However, the performance evaluation does not consider a real video traffic and as a result the user quality of experience (QoE) is not evaluated either. More recently, the work in [5] defined another objective function based on the remaining energy. Results show that energy consumption is better distributed among nodes. Simulations are made using H. 264 multimedia trace [6]. However, the user QoE is not considered. Moreover, H. 264 compression is not adapted to low-power video sensors [7].

Network lifetime can be maximised by minimising the radio activity characterised by its high power consumption in the transmit, receive as well as idle listening periods. In order to save energy, radio duty cycling (RDC) protocols are suggested to switch off the radio as much as possible. With respect to visual data, compression is also required before transmission to limit the amount of data to route and hence limit power consumption. In this paper, we investigate the feasibility of real video streaming using RPL. We mainly address radio duty cycling impact on the transmission of low complexity compressed images. As opposed to [4] and [5], we consider a compression technique that is more adapted to LLNs. Moreover, we do not restrict our evaluation to traditional QoS metrics such as packet delivery ratio. Rather, we assess the quality of received video using QoE metrics namely PSNR (Peak Signal to Noise Ratio) and SSIM (Structural SIMilarity).

To the best of our knowledge, this is the first work that evaluates video transmission using RPL where both QoS and QoE metrics are considered along with a low compression technique suitable to LLNs. The followed compression method is presented in Section II along with the used QoE metrics. The simulation scenario is detailed in Section III and the obtained results are discussed in Section IV before concluding.

\section{LOW COMPLEXITY COMPRESSION AND QOE METRICS}

In order to suite the constrained nature of processing resources of low-power motes, we made use of a low complexity image compression provided by SenseVid [8], a video transmission and evaluation tool that considers WVSN specific characteristics. First, each captured image is decomposed into blocks of $8 \times 8$. Then, a fast pruned DCT (binDCT-C [9]) is applied on each block with a triangular pattern [10]. Only coefficients located at the upper left triangle of side length $\rho \leq 8$ are considered. The resulting DCT block coefficients are 


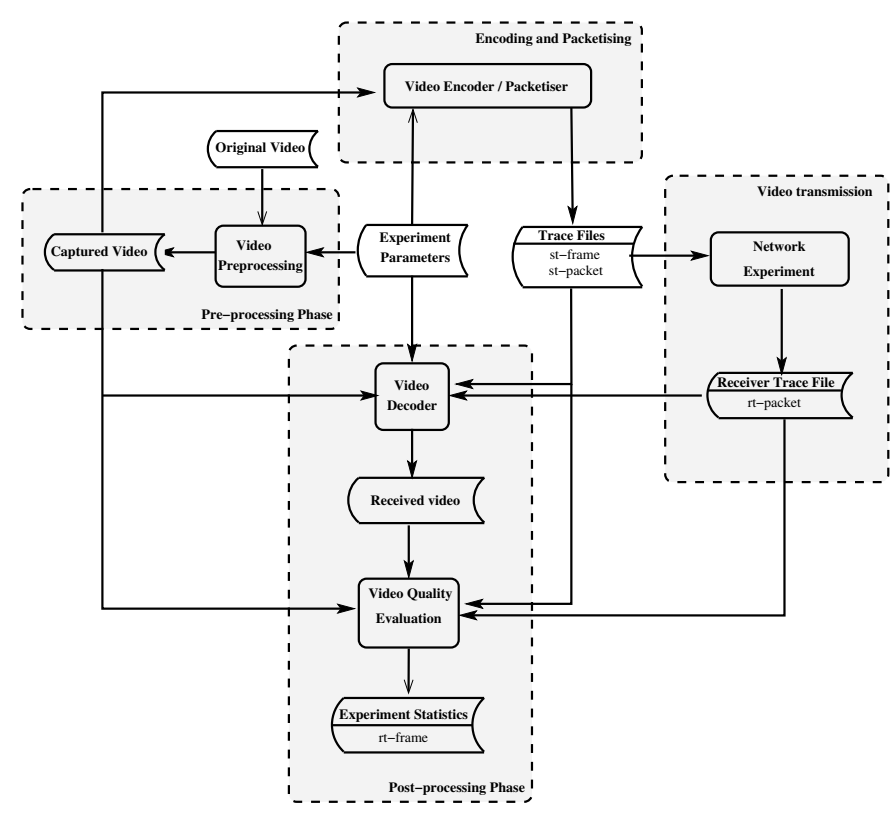

Fig. 1: SenseVid Architecture (redrawn from [8])

quantised using the JPEG standard quantisation matrix. Tradeoff between quality level and compression rate can be obtained by selecting a proper quality factor $(\mathrm{QF})$ that allows adjusting the quantisation matrix values. An image visual quality ranges from the poorest $(Q F=1)$ to the best quality $(Q F=100)$. We set $Q F=8$ in our experiments allowing for a compression of about 1 bpp when $\rho=8$ (default value). The obtained block is then linearised using the traditional zigzag scan. Finally, exponential-Golomb code [11] is applied as a lossless entropy encoding.

SenseVid, as shown in Figure 1, follows the main principle of EvalVid [12] where the interface with the experiment is insured by the use of traffic trace files. According to the user parameters, two trace files are generated. The frame trace file that includes information on the encoded images and the sender trace file gives the list of data packets to be sent by the source. The sink records the received data packets in a receiver trace file. Based on the trace files, SenseVid reconstructs the video received images allowing their subjective assessment by the user. Moreover, it generates QoE video related metrics. PSNR and SSIM metrics allow to assess the quality of both the encoded video before transmission (reference PSNR or SSIM) and the received video with respect to the initial lossless encoded video. The PSNR between the sent and the received, possibly distorted video frame is computed using :

$$
P S N R=20 \log \frac{V_{\text {peak }}}{M S E}
$$

where $M S E$ is the mean square error and $V_{\text {peak }}$ is the maximum possible pixel value. The SSIM metric is computed as follows :

$$
S S I M=\frac{2 \mu_{x} \mu_{y}+C_{1}}{\mu_{x}^{2}+\mu_{y}^{2}+C_{1}} \times \frac{2 \sigma_{x} \sigma_{y}+C_{2}}{\sigma_{x}^{2}+\sigma_{y}^{2}+C_{2}} \times \frac{\sigma_{x y}+C_{2} / 2}{\sigma_{x} \sigma_{y}+C_{2} / 2}
$$

where $x$ and $y$ are two non negative image signals, $\mu_{x}, \sigma_{x}$ and $\mu_{y}, \sigma_{y}$ are the mean and standard deviation of $x$ and $y$ respectively. $\sigma_{x y}$ is the sample cross-covariance between $x$ and y. $C_{1}$ and $C_{2}$ are set respectively to 6.5025 and 58.5225 .

\section{Performance Evaluation Scenario}

In order to carry out our performance evaluation of visual data transmission in a constrained network, we made use of Cooja [13], the Contiki network simulator. As a routing protocol, we used RPL implementation provided in the Contiki IPv6 network stack. We chose ContikiMAC [14] as the radio duty cycling protocol. When a sender has to transmit a unicast packet, it repeatedly sends that packet during a predefined period or till an acknowledgement (ACK) is received. For each of its neighbours, the sender stores the timing of received ACKs. This allows the sender to start transmitting a unicast packet just before the destination wakes up. Duty cycling can be tuned using Channel Check Rate (CCR) parameter defined as the frequency a node will listen to the medium to eventually receive data from its neighbours. When some activity is detected the node stays awake to receive data ; otherwise the node goes back to sleep mode for another duty cycling period.

We considered a square sensor field of size $90 \times 90 \mathrm{~m}^{2}$ where 16 static sensor nodes (Tmote Sky) are deployed in a grid topology. The sink is located at the upper left corner and the video source is located the lower right corner. Transmission range is set to 50 meters resulting in 8 as a maximum node degree (neighbours) and 3 as the minimum number of hops from source to the sink. Tmote Sky motes [15] are equipped with chipcon CC2420 radio (IEEE 802.15.4 compliant) for wireless communications. The radio power dissipation is $75.6 \mathrm{~mW}$, $82.8 \mathrm{~mW}$ and $4.32 \mathrm{~mW}$ for respectively active, listen, and idle states. The Contiki provided powertrace tool is used to estimate power consumption. Performance evaluation metrics are averaged over at least 10 simulations. Main experiments parameters are summarised in Table I.

We modified the Contiki RPL-UDP server and client code to allow the use of trace files generated by SenseVid. The sender transmits data packets with a maximum payload of 112 Bytes following the sender trace file. The sink records the list of received packets in the receiver trace file. The sender trace file results from the encoding of gray scale captured images from the hall monitor video clip [16] down-sampled to half QCIF resolution. Instead of 25 frames per second, the frame frequency capture (FFC) is set to values ranging from 10 to 60 frames per minute. Sending video sequences in a 25 frames per second is simply unfeasible due to the limited resources of the considered Tmote Sky sensor motes in our topology.

\section{Simulation Results}

We first study the impact of varying the channel check rate of sensor nodes on packets delivery ratio, the quality 
TABLE I: Simulation Parameters

\begin{tabular}{|l|l|}
\hline Area dimensions & $90 \times 90$ \\
Number of sensors & $16-$ grid $4 \times 4$ \\
Sensors type & Tmote Sky \\
Packet maximum payload & 112 Bytes \\
Transport protocol & UDP \\
Routing protocol & RPL (IPv6) \\
RPL DiO min/max interval & $4 \mathrm{~s} / 17.5 \mathrm{~min}$ \\
RPL objective function & ETX \\
MAC & CSMA / link-layer bursts \\
Radio Duty Cycling & ContikiMAC, NullRDC \\
Channel check rates $(\mathrm{Hz})$ & $8,16,32,64,128$ \\
Physical & IEEE 802.15 .4 \\
Radio active power & $75.6 \mathrm{~mW}$ \\
Radio idle power & $4.32 \mathrm{~mW}$ \\
Radio listening power & $82.8 \mathrm{~mW}$ \\
Video duration & 12 seconds \\
Frame resolution & $88 \times 72$ \\
Frame frequency capture (FFC) & $10,15 \ldots 55,60$ frames/mn \\
Number of captured Images & $2,3, \ldots 11,12$ \\
GOP Coef. & $0-$ all images are intra-coded \\
Quality Factor & 8 \\
DCT & Triangular BIN DCT \\
DCT triangle side length $\rho$ & $3,6,8$ \\
Entropy coder & Exponential-Golomb (EG) \\
\hline
\end{tabular}

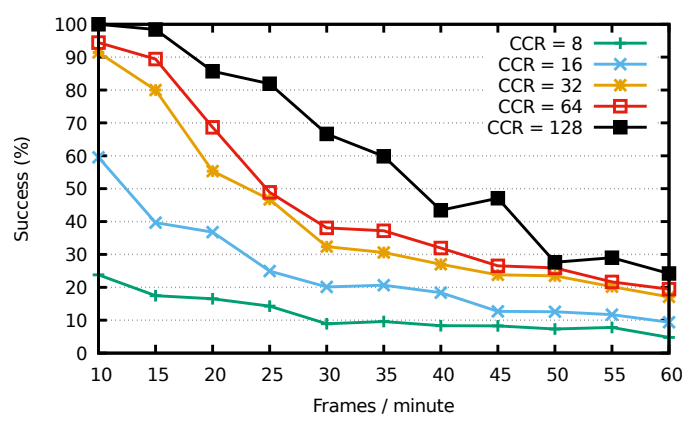

Fig. 2: Success ratio.

of reconstructed images by the sink as well as the amount of consumed energy. The frequency of images capture is increased in order to estimate the highest FFC for which an acceptable images quality is obtained. Afterwards, in an attempt to further decrease the amount of data to transmit, we diminish the DCT triangular side length $(\rho)$.

\section{A. Impact of Duty Cycling}

Figure 2 plots the obtained ratio of successfully delivered packets to the sink when increasing the FFC from 10 to 60 images per minute for different duty cycling settings. We can see that the higher the CCR, the higher the packets delivery ratio. This ratio decreases when increasing the number of captured frames. Almost all packets are received when CCR is set to 128 for 10 and 15 frames sent per minute. However, when the CCR is lowered to 8 , the success ratio falls down to, for instance, less than $20 \%$ for 15 frames per minute.

Figure 3 shows the average PSNR and SSIM of received images. In general, an acceptable quality is achieved with at least a PSNR of $20 \mathrm{~dB}$. With the highest CCR, we are only able to obtain a fair image quality for a maximum rate of 35

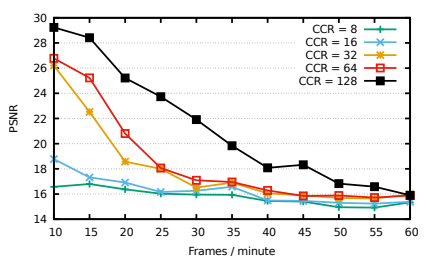

(a) PSNR (dB).

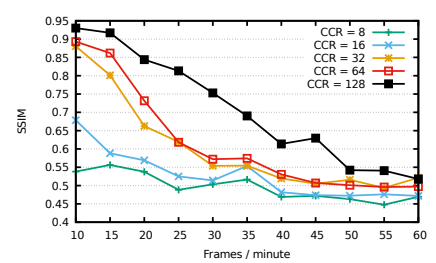

(b) SSIM
Fig. 3: Quality of received images.

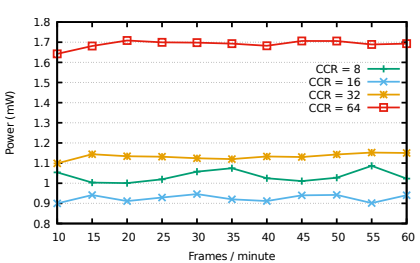

(a) Overall power $(\mathrm{mW})$.

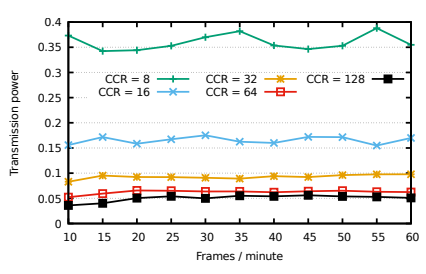

(b) Transmission power $(\mathrm{mW})$.
Fig. 4: Power consumption.

frames per minute. Lower CCR values exhibit very poor image quality even with only 10 frames sent per minute.

In order to get more insight on the observed delivery ratio, we plot the mean consumed power based on averages over time and individual power consumption records of the different nodes in the network. Figure 4a plots the overall depleted power as the number of sent frames per minute is increased. The case $C C R=128$ where more than $21 \mathrm{~mW}$ of overall power is measured, is omitted for clarity. Figure $4 \mathrm{~b}$ plots the depleted power due to radio transmission activities. The main observation is that power consumption does not depend on the transmission rate (FFC). Figure $4 \mathrm{a}$ shows that the more the $\mathrm{CCR}$, the more consumed energy except for $C C R=8$. In fact, a CCR set to 16 triggers less energy consumption compared to a CCR of 8 . This is due to the fact that in the later case, the number of retransmissions is higher as confirmed by Figure $4 \mathrm{~b}$. We note that the highest depleted transmission power corresponds to a CCR set to 8 which attests the highest experienced transmission attempts.

Figure 5 plots the depleted power due to radio transmission activities as time evolves for an FFC of 10 and 40 frames per minute. Recall that the video lasts 12 seconds and the frames are captured as required by the FFC parameter within this duration. Frames transmission is started approximately around 8 seconds. We can observe that the higher the CCR, the lower the interval where power consumption is maximised and the lower the consumed power. We can see that for the lowest CCR, transmissions last longer and power consumption achieves much higher values, more than $1.8 \mathrm{~mW}$ for $F F C=40$.

\section{B. Impact of Reducing the Bit Rate}

The high losses experienced in the previous section are mainly due to the amount of data that the source has to 


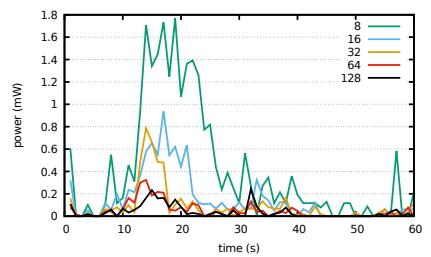

(a) $F F C=10$

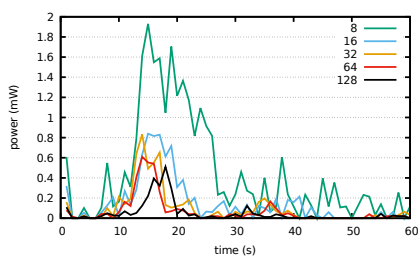

(b) $F F C=40$
Fig. 5: Transmission power

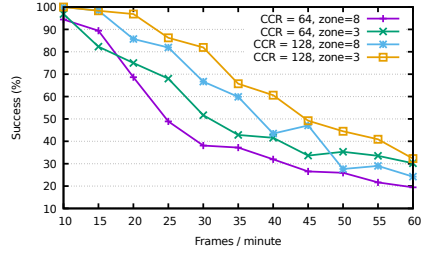

(a) Success ratio.

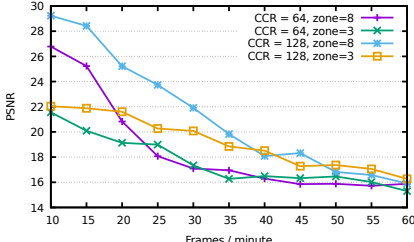

(b) PSNR.
Fig. 6: Impact of reducing the bit rate

transmit in a limited time window. In order to reduce the number of lost packets, we performed simulations where only a subset of the upper triangle of each block are compressed and transmitted. This is done by setting the triangle side length $(\rho)$ to 3 instead of 8 which allows to reduce the bit rate from 0.99 to $0.52 \mathrm{bpp}$. The reference PSNR however decreases from about $29 \mathrm{~dB}$ to about $22 \mathrm{~dB}$. It is worth to mention that power consumed by the radio module is not influenced by decreasing the bit rate. The source, however, can gain some energy due to less computing tasks related to the compression of captured images. We note according to Figure 6a that the delivery ratio is increased when $\rho$ is set to 3 since the amount of data to send per time unit is reduced. We, for instance, observe up to $61 \%$ success ratio improvement for a capture rate of 50 frames per minute when $C C R=128$.

Although packets delivery ratio is improved when decreasing the triangle length side, we observe that the quality of received video is not enhanced as shown by Figure 6b. This is due to the fact that the reference PSNR is lowered when the number of considered coefficients in each block is reduced. We also can note that images quality becomes almost equivalent when the transmission rate achieves a given threshold. This rate corresponds to 40 and 25 frames per minute for a CCR of 128 and 64 respectively.

\section{CONCLUSION}

In this paper, we investigated the feasibility of visual data transmission using RPL. We adopted a low complexity compression to cope with limited resources at the source node. We mainly proceed by varying the duty cycling in order to assess its effect on power consumption as well as the overall performances of the visual data transmission. In addition to traditional QOS metrics, we used QoE metrics such as PSNR and SSIM to evaluate the quality of received images.
We showed that RPL allows to transmit images at low rates up to 35 frames per minute when images are encoded using our low power technique. As a result, RPL is far from being able to handle real time video transmission. Substantial effort will be required on at least two fronts. On the one hand, low power compression techniques with limited bit rate have to be developed. On the other hand, much effort has to be undertaken to adapt RPL as well as the MAC/RDC layer to the high data rate required by visual data. In fact, our results proved that the radio duty cycle has a significant effect in the quality of received images. However, maximising the radio activity affects the network lifetime as more energy is depleted.

\section{REFERENCES}

[1] B. Tavli, K. Bicakci, R. Zilan, and J. M. Barcelo-Ordinas, "A survey of visual sensor network platforms," Multimedia Tools and Applications, vol. 60, no. 3, pp. 689-726, 2012.

[2] R. Alexander, A. Brandt, J. Vasseur, J. Hui, K. Pister, P. Thubert, P. Levis, R. Struik, R. Kelsey, and T. Winter, "RPL: IPv6 Routing Protocol for Low-Power and Lossy Networks," RFC 6550, Mar. 2012. [Online]. Available: https://rfc-editor.org/rfc/rfc6550.txt

[3] D. S. J. De Couto, D. Aguayo, J. Bicket, and R. Morris, "A highthroughput path metric for multi-hop wireless routing," in Proceedings of the 9th ACM International Conference on Mobile Computing and Networking (MobiCom '03), San Diego, California, September 2003.

[4] S. A. Alvi, G. A. Shah, and W. Mahmood, "Energy efficient green routing protocol for internet of multimedia things," in 2015 IEEE Tenth International Conference on Intelligent Sensors, Sensor Networks and Information Processing (ISSNIP). IEEE, 2015, pp. 1-6.

[5] F. Mortazavi and M. Khansari, "An energy-aware rpl routing protocol for internet of multimedia things," in Proceedings of the International Conference on Smart Cities and Internet of Things, ser. SCIOT '18. New York, NY, USA: ACM, 2018, pp. 11:1-11:6. [Online]. Available: http://doi.acm.org/10.1145/3269961.3269965

[6] P. Seeling, M. Reisslein, and B. Kulapala, "Network performance evaluation using frame size and quality traces of single-layer and twolayer video: A tutorial," Communications Surveys Tutorials, IEEE, vol. 6, no. 3, pp. 58-78, Third 2004.

[7] B. A. B. Sarif, M. Pourazad, P. Nasiopoulos, and V. C. M. Leung, "A study on the power consumption of H.264/AVC-based video sensor network," International Journal of Distributed Sensor Networks, vol. 11, no. 10, p. 304787, 2015. [Online]. Available: https://doi.org/10.1155/2015/304787

[8] M. Maimour, "Sensevid: A traffic trace based tool for qoe video transmission assessment dedicated to wireless video sensor networks," Simulation Modelling Practice and Theory, vol. 87, pp. 120-137, September 2018.

[9] J. Liang and T. D. Tran, "A fast multiplierless approximations of the dct with the lifting scheme," IEEE Transactions on Signal Processing, vol. 49, no. 2, pp. 3032-3044, December 2001.

[10] A. Mammeri, A. Khoumsi, D. Ziou, and B. Hadjou, "Modeling and adapting jpeg to the energy requirements of VSN," in Computer Communications and Networks, 2008. ICCCN '08. Proceedings of 17th International Conference on, 2008, pp. 1-6.

[11] J. Teuhola, "A compression method for clustered bit-vectors," Information processing letters, vol. 7, no. 6, pp. 308-311, 1978.

[12] J. Klaue, B. Rathke, and A. Wolisz, "Evalvid - a framework for video transmission and quality evaluation," in In Proc. of the 13th International Conference on Modelling Techniques and Tools for Computer Performance Evaluation, 2003, pp. 255-272, ttp://www.tkn.tuberlin.de/research/evalvid/.

[13] I. Romdhani, M. Qasem, A. Y. Al-Dubai, and B. Ghaleb, "Cooja simulator manual," Edinburgh Napier University, Tech. Rep., 2016.

[14] A. Dunkels, "The contikimac radio duty cycling protocol," Swedish Institute of Computer Science, Tech. Rep., 2011.

[15] Tmote Sky Datasheet, Moteiv Corporation. [Online]. Available: http://sentilla.com/files/pdf/eol/tmote-sky-datasheet.pdf

[16] "Yuv video sequences." [Online]. Available: http://trace.eas.asu.edu/yuv/index.html 